\title{
Relapse of aseptic meningitis induced by ipilimumab and nivolumab therapy for metastatic renal cell carcinoma: A case report
}

\author{
DAI TAKAMATSU, NOBUKI FURUBAYASHI, TAKAHITO NEGISHI, KOSUKE IEIRI, \\ TOMOHIRO INOUE, KEIJI TSUKINO and MOTONOBU NAKAMURA
}

Department of Urology, National Hospital Organization Kyushu Cancer Center, Fukuoka 811-1395, Japan

Received June 6, 2019; Accepted September 12, 2019

DOI: $10.3892 /$ mco.2019.1929

\begin{abstract}
The combined immunotherapy of nivolumab and ipilimumab causes a variety of autoimmune-related adverse events (irAEs). The current report details a 70-year-old woman with clear cell renal cell carcinoma metastasis in the lung. Two weeks after two courses of treatment, the patient complained of headache, dizziness and nausea. Cerebrospinal fluid (CSF) analysis revealed an elevated protein level of $195 \mathrm{mg} / \mathrm{dl}$ and a significantly elevated white blood cell (WBC) count of $830 / \mathrm{mm}^{3}$ (lymphocytes, $825 / \mathrm{mm}^{3}$; neutrophils, $5 / \mathrm{mm}^{3}$ ). The results excluded malignancy and infection. The patient was diagnosed with aseptic meningitis and was administered intravenous prednisolone ( $1 \mathrm{mg} / \mathrm{kg} /$ day $)$. On the 49 th day of the $2 \mathrm{nd}$ course of treatment, no recurrence of clinical symptoms was exhibited during maintenance oral steroid treatment (prednisolone $10 \mathrm{mg} /$ day) and CSF analysis revealed that the WBC count had dropped to $44 / \mathrm{mm}^{3}$ (lymphocytes only). Therefore, the 3rd course of treatment was readministered the next day. After two weeks, the patients again complained of nausea, anorexia and fatigue. CSF analysis demonstrated that the WBC count was not increased from the result obtained previously. However, brain MRI scans revealed the mild diffuse enlargement of the pituitary and endocrine system tests revealed reduced adrenocorticotropic hormone (ACTH; $2.0 \mathrm{pg} / \mathrm{ml}$ ) and cortisol $(1.12 \mu \mathrm{g} / \mathrm{dl})$ levels. The patient was diagnosed with isolated ACTH deficiency and oral hydrocortisone was administered after prednisolone cessation. On the 25th day of the 3rd course of treatment, the patient complained of headache and anorexia. CSF examination revealed that the WBC count had increased a second time $\left(53 / \mathrm{mm}^{3}\right.$; lymphocytes only) and laboratory data revealed hepatic dysfunction. The patient was then diagnosed
\end{abstract}

Correspondence to: Dr Nobuki Furubayashi, Department of Urology, National Hospital Organization Kyushu Cancer Center, Notame 3-1-1, Minami-ku, Fukuoka 811-1395, Japan

E-mail: furubayashi-jua@umin.ac.jp

Key words: renal cell carcinoma, ipilimumab, nivolumab, meningitis, immune-related adverse events with relapse of aseptic meningitis and liver dysfunction. While continuing oral hydrocortisone treatment, the administration of intravenous prednisolone was started. The observed liver dysfunction and aseptic meningitis gradually improved. The current report may be useful for avoiding delays in the diagnosis and treatment of this life-threatening and uncommon irAE, in which CSF examinations are useful for diagnosis and management.

\section{Introduction}

In recent years, combination therapy with nivolumab, a programmed death 1 (PD-1) immune checkpoint inhibitor antibody, and ipilimumab, an anti-cytotoxic T-lymphocyte-associated antigen 4 (CTLA-4) antibody, have demonstrated clinical efficacy in the treatment of metastatic RCC (mRCC) patients (1). These results led to the United State Food and Drug Administration approving the combination of ipilimumab and nivolumab in treatment-naïve patients with intermediate- or poor-risk disease according to the International Metastatic Renal Cell Carcinoma Database Consortium (IMDC) criteria in April 2018 (2,3). In Japan, this combination therapy has also been approved since August 2018. However, it is often associated with a wide variety of immune-related adverse events (irAEs) that can affect almost any organ site $(1,4)$.

We herein report a patient with metastatic renal cell carcinoma who developed the uncommon irAE of aseptic meningitis as well as isolated ACTH deficiency and liver dysfunction during ipilimumab and nivolumab therapy.

\section{Case report}

A 70-year-old Japanese woman was referred to our institution for the evaluation of a right renal tumor that had been detected by abdominal ultrasonography at a screening examination in July 2018. She had a history of hypertension. She was diagnosed with right renal cell carcinoma (cT1bNOM0) by computed tomography (CT) and underwent right nephrectomy in the same month. Two months later, multiple lung metastases were observed by CT (Fig. 1A). Therefore, she was diagnosed as 'intermediate risk' according to the IMDC 


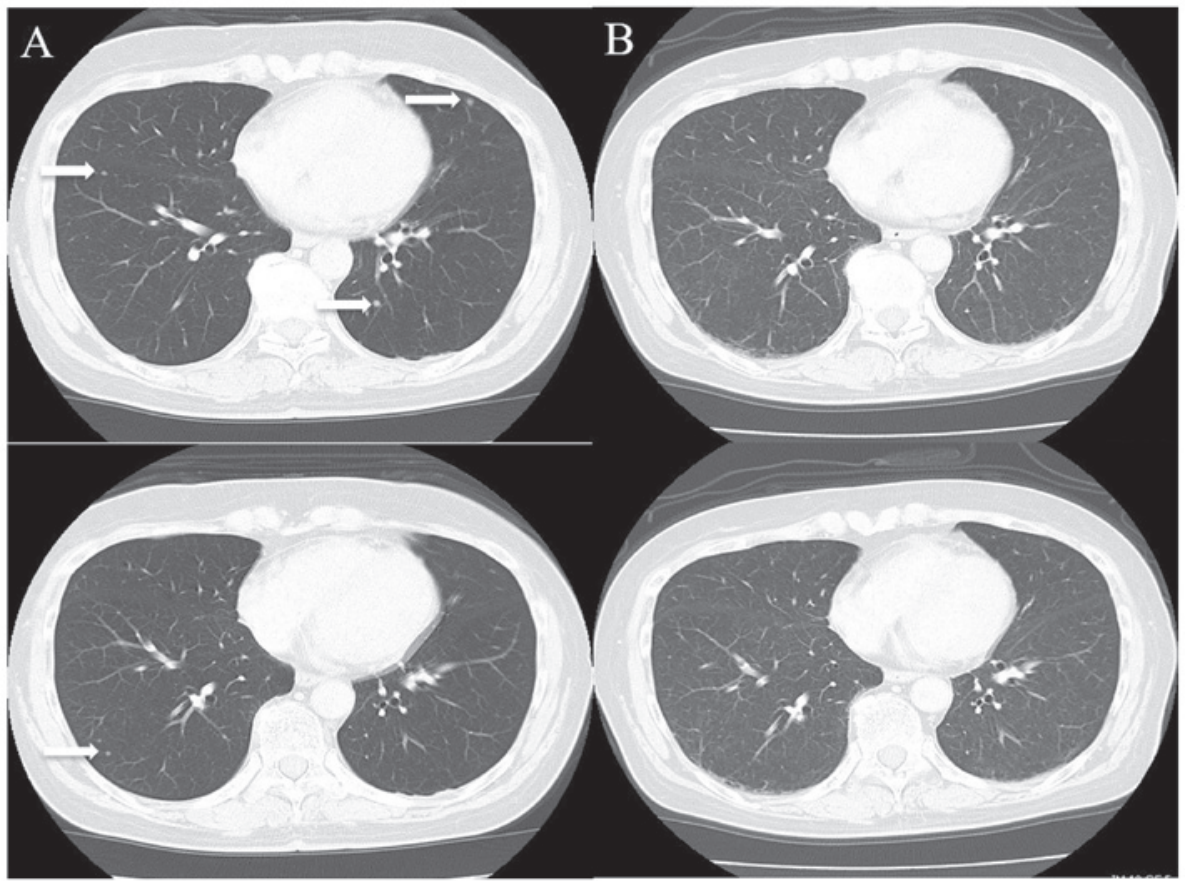

Figure 1. Chest CT. (A) Chest CT revealed metastatic lung tumors in the bilateral lobe prior to ipilimumab and nivolumab combinational therapy (white arrow). (B) Following the 3rd course of therapy, CT images revealed that the lung metastasis had disappeared.
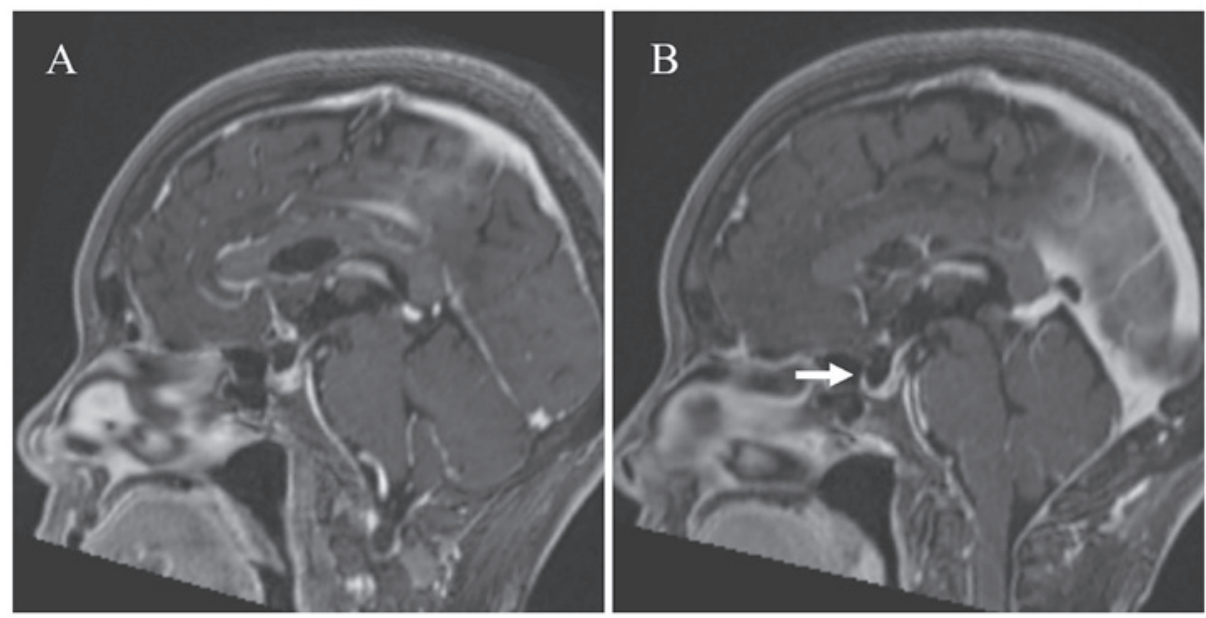

Figure 2. Brain MRI. (A) A sagittal section of a contrast-enhanced T1-weighted brain MRI scan revealed no abnormality. (B) A sagittal section of a contrast-enhanced T1-weighted brain MRI scan presented mild diffuse enlargement of the pituitary (white arrow).

criteria [she had one prognostic factor $(<1$ year since the diagnosis)].

Combination therapy (once every 3 weeks, intravenously) of ipilimumab (1 mg/kg) and nivolumab (240 mg/body) was administered as the first-line therapy in September 2018. On the 14th day of the 2nd course, she complained of nonspecific clinical symptoms, such as headaches, dizziness and nausea, and was admitted to our hospital. However, she did not complain of or develop any other specific clinical features pertaining to the central nervous system. She also did not report any neck stiffness. Brain magnetic resonance imaging (MRI) was preformed, but there were no brain metastases or any findings suggestive of encephalitis or meningitis (Fig. 2A). However, meningitis could not be ruled out clinically, so a cerebrospinal fluid (CSF) test was performed.
The examination of the CSF revealed normal glucose levels but an elevated protein level at $195 \mathrm{mg} / \mathrm{dl}$ and a significantly elevated white blood cell (WBC) count of $830 / \mathrm{mm}^{3}$ (lymphocytes $825 / \mathrm{mm}^{3}$, neutrophils $5 / \mathrm{mm}^{3}$; Table I). In addition, CSF cytology showed no malignant cells, and the CSF analysis was negative for bacteria and viruses (cytomegalovirus, herpes simplex virus, varicella-zoster virus and human herpes virus type 6). Based on these results, she was diagnosed with aseptic meningitis induced by ipilimumab and nivolumab therapy, and prednisolone $(1 \mathrm{mg} / \mathrm{kg} / \mathrm{day})$ was started intravenously. After a few days, her clinical symptoms improved rapidly, as did the CSF analysis findings, so prednisolone was tapered gradually. On the 49th day of the 2nd course, she had no recurrence of clinical symptoms on maintenance oral steroid treatment (prednisolone $10 \mathrm{mg} / \mathrm{day}$ ), 
Table I. CSF analysis and blood glucose data of the case study.

\begin{tabular}{lcccccc}
\hline CSF examination & Reference value & C2D14 & C2D23 & C2D49 & C3D10 & C3D25 \\
\hline Glucose, $\mathrm{mg} / \mathrm{dl}$ & $45-80$ & 46 & 74 & 67 & 66 & 66 \\
Protein, $\mathrm{mg} / \mathrm{dl}$ & $<45$ & 195 & 97 & 41 & 52 & 48 \\
WBC, $\mathrm{n} / \mathrm{mm}^{3}$ & $<5$ & 830 & 217 & 44 & 34 & 53 \\
Lymphocyte, $\mathrm{n} / \mathrm{mm}^{3}$ & 0 & 825 & 215 & 44 & 34 & 53 \\
Neutrophil, $\mathrm{n} / \mathrm{mm}^{3}$ & 0 & 5 & 2 & 0 & 0 & 0 \\
Blood glucose, $\mathrm{mg} / \mathrm{dl}$ & $73-109$ & 106 & - & - & 148 & 141 \\
Blood to CSF glucose ratio & & 0.43 & - & - & 0.45 & 0.47 \\
\hline
\end{tabular}

CSF, cerebrospinal fluid; C, course; D, day; WBC, white blood cell.

Table II. Patient laboratory data.

\begin{tabular}{|c|c|c|c|c|c|c|c|}
\hline Laboratory data & Reference value & C3D10 & C3D15 & C3D25 & C3D27 & C3D29 & C3D50 \\
\hline WBC, n & $3,300-8,600 / \mu 1$ & 11,000 & 9,000 & 9,170 & 8,850 & 19,030 & 16,920 \\
\hline Eosino $(\%)$ & $0.4-8.6$ & 0.5 & 1.3 & 0.3 & 0.6 & 0.1 & 0.2 \\
\hline AST, U/1 & $13-30$ & 35 & 50 & 131 & 155 & 69 & 20 \\
\hline ALT, U/1 & $10-42$ & 41 & 57 & 204 & 244 & 176 & 44 \\
\hline $\mathrm{Na}, \mathrm{mmol} / \mathrm{l}$ & $138-145$ & 133 & 122 & 127 & 131 & 134 & - \\
\hline CRP, mg/dl & $0-0.14$ & 3.12 & 6.45 & 0.36 & 0.27 & $<0.07$ & - \\
\hline ACTH, pg/ml & $7.2-63.3$ & 15.7 & 2 & $<1.5$ & - & - & - \\
\hline Cortisol, $\mu \mathrm{g} / \mathrm{dl}$ & $6.24-18.0$ & 3.18 & 1.12 & 38.1 & - & - & - \\
\hline $\mathrm{TSH}, \mu \mathrm{IU} / \mathrm{ml}$ & $0.49-4.67$ & - & 0.31 & 2.5 & - & - & - \\
\hline F-T4, ng/ml & $0.71-1.85$ & - & 0.91 & 0.89 & - & - & - \\
\hline PRL, ng/ml & 4.29-13.69 & - & 60.5 & - & - & - & - \\
\hline $\mathrm{LH}, \mathrm{mIU} / \mathrm{ml}$ & $0.79-5.72$ & - & 8.08 & - & - & - & - \\
\hline FSH, mIU/l & $2.00-8.30$ & - & 30.54 & - & - & - & - \\
\hline
\end{tabular}

Certain data are not provided, as these parameters did not require follow-up at these stages. WBC, white blood cell; Eosino, eosinophil ratio; AST, aspartate aminotransferase; ALT, alanine aminotransferase; CRP, C-reactive protein; ACTH, adrenocorticotropic hormone; TSH, thyroid-stimulating hormone; F-T4, thyroxine; PRL, prolactin; LH, luteinizing hormone; FSH, follicle-stimulating hormone; C, course; D, day.

and a CSF analysis showed that the WBC count had dropped to $44 / \mathrm{mm}^{3}$ (only lymphocytes; Table I). After delivering a sufficient explanation of the risks and benefits to the patient, the $3 \mathrm{rd}$ course of the combination therapy was readministered the next day.

Two weeks later, she complained of nausea, anorexia and fatigue. A relapse of meningitis was suspected, but the CSF showed that the WBC count was not marked elevated compared to before (Table I). Although CT was performed to investigate the cause, no abnormal findings were found. However, all multiple lung metastases had disappeared, and a complete response was considered to have been achieved (Fig. 1B). The MRI of the brain showed mild diffuse enlargement of the pituitary (Fig. 2B). Her laboratory data showed a low sodium value $(122 \mathrm{mmol} / \mathrm{l})$. Based on the clinical symptoms, examination values and MRI findings, adrenocorticotropic hormone (ACTH) deficiency was suspected, and the administration of oral hydrocortisone $20 \mathrm{mg} /$ day $\left(\right.$ Cortril $^{\circledR}$; morning $15 \mathrm{mg}$, evening $5 \mathrm{mg}$ ) was started after stopping prednisolone.
Two days later, her symptoms dramatically improved. The results of the endocrine system tests revealed that her ACTH $(2.0 \mathrm{pg} / \mathrm{ml})$ and cortisol $(1.12 \mu \mathrm{g} / \mathrm{dl})$ levels were lower than normal. The levels of other anterior pituitary hormones (prolactin, luteinizing hormone, follicle-stimulating hormone, thyroid-stimulating hormone) were not low (Table II). Based on these findings, the diagnosis was isolated ACTH deficiency induced by combination therapy of ipilimumab and nivolumab.

On the 25th day of the 3rd course, she complained of headache and anorexia. The CSF examination showed that the WBC count had increased again $\left(53 / \mathrm{mm}^{3}\right.$, only lymphocytes), and her laboratory data showed hepatic dysfunction and elevated aspartate aminotransferase at $155 \mathrm{U} / 1$ and alanine aminotransferase at $244 \mathrm{U} / 1$ (Tables I and II). She had no history of infection with hepatitis $B$ and $C$ virus, and abdominal echography showed no remarkable findings in her liver. She was diagnosed with liver dysfunction and relapse of aseptic meningitis induced by combination therapy of ipilimumab and nivolumab. 
While continuing the administration of oral hydrocortisone $20 \mathrm{mg} / \mathrm{day}$, the administration of intravenous prednisolone (total $1 \mathrm{mg} / \mathrm{kg} /$ day) was started additionally. The liver dysfunction and aseptic meningitis gradually improved (Table II). Three months later, CT showed that the multiple lung metastases had not reappeared. However, she had been taking oral hydrocortisone $20 \mathrm{mg}$ /day for isolated ACTH deficiency and prednisolone $2.5 \mathrm{mg} /$ day for liver dysfunction. Therefore, the immunotherapy was not restarted.

\section{Discussion}

Anti-CTLA-4 antibody and anti-PD-1 antibody have different working points and mechanisms of activity, and their combination brings about a synergetic effect on the tumor reduction effect and overall survival $(1,5)$. However, the frequency and severity of irAEs is also synergistically affected. Although the patient backgrounds differed between these studies, grade 3 or 4 treatment-related AEs occurred in $18.7 \%$ of patients treated with nivolumab alone for mRCC (6), whereas such AEs occurred in $45.7 \%$ of patients treated with nivolumab plus ipilimumab for mRCC (1). The most frequently reported irAEs in the RCC population are skin events (59.6\% of patients), endocrine events (40.4\% of patients), gastrointestinal events ( $44.7 \%$ of patients), hepatic events $(27.7 \%)$, renal events $(12.8 \%)$ and pulmonary events (10.6\%) (7). Neurologic side-effects are rare but include cases of immune polyneuropathies, Guillain Barré syndrome, myasthenia gravis, posterior reversible encephalopathy syndrome, aseptic meningitis, enteric neuropathy, transverse myelitis as well as immune encephalitis (8). In a pooled analysis of nearly 1,500 patients with melanoma treated with ipilimumab, the incidence of neurologic irAEs was $0.1 \%(9,10)$.

We herein report a 70 -year-old woman with mRCC receiving combination nivolumab and ipilimumab therapy who developed aseptic meningitis, isolated ACTH deficiency and hepatic dysfunction that were probably induced by an auto-immune related mechanism. As meningitis and encephalitis were suspected based on clinical symptoms, lumber puncture proved extremely helpful for making a diagnosis because there were no findings on brain MRI. CSF abnormalities with an elevated proteins level associated with lymphocytic pleocytosis supported the presence of inflammatory-mediated disease. General bacterial meningitis was initially excluded because of the lymphocyte-dominated findings on CSF. Tuberculous meningitis has also been reported to have lymphocyte-dominated findings. However, in modern Japan, tuberculosis infection itself is so rare that it was not considered as a differential diagnosis. A CSF analysis was the most useful examination for supporting the relationship between the aseptic meningitis status and the response to steroid treatment. Aseptic meningitis fortunately responded well to high-dose steroid therapy, and her irAEs also improved to Grade 1 promptly. The early improvement of the patient's symptoms and CSF analysis findings by steroid treatment ruled out tuberculous meningitis. There were no sequelae, and the prednisolone dose was able to be reduced to $10 \mathrm{mg}$ /day within 1 month after the start of steroid therapy.

Whether or not to resume immune checkpoint inhibitor treatment after neurological recovery is a matter of debate, and a careful analysis of the risks and benefits should be done on a case by case basis. The therapeutic effect was a complete response after only 2 courses. However, the durability of the response to this combination therapy for mRCC still remains unclear and this combination immunotherapy is only allowed in the first-line setting for mRCC in Japan. Steroid treatment therapy for this irAE foutunately demonstrated a good response, so the combination therapy was re-administered after both the risks and benefits were sufficiently explained to the patient. However, isolated ACTH deficiency occurred with the oral administration of steroids. Furthermore, relapse of aseptic meningitis and liver dysfunction also occurred. An autopsy analysis of the pituitary glands of patients with cancer treated by CTLA-4 blockade described type II and IV hypersensitivity reactions and a strong CTLA-4 expression in the pituitary gland (11). Therefore, this hypophysitis with pituitary enlargement hypothetically could have been caused by type II hypersensitivity reactions, such as complement activation and infiltration with macrophages and phagocytosis, and type IV hypersensitivity reactions, such as infiltration with autoreactive $\mathrm{T}$ lymphocytes. Although the study was conducted in a melanoma population, it was reported that approximately $40 \%$ of patients with advanced melanoma who received nivolumab combined with ipilimumab in clinical trials discontinued treatment because of AEs. However, the efficacy outcomes seemed similar between patients who discontinued nivolumab plus ipilimumab treatment because of AEs during the induction phase and those who did not discontinue the treatment because of AEs (12). In the present case, re-administration of nivolumab and ipilimumab therapy occurred not only other irAEs but also relapse of aseptic meningitis. Therefore, we decided to resume immunotherapy when the disease progressed.

No standard treatment has yet been defined for neurological irAEs. A commonly used protocol is oral prednisone at roughly $1 \mathrm{mg} / \mathrm{kg} /$ day. A neurological irAE management algorithm has been proposed $(5,8)$. However, further studies are necessary in order to establish the precise framework regarding the dose, timing of tapering and administration period of steroid treatment. In the present case, a longer tapering period for steroids might have been needed in order to prevent recurrent irAEs. Indeed, it was reported that neurological irAEs are not always reversible (13), highlighting the importance of optimizing treatment regimens.

With the increasing use of immune checkpoint inhibitors in cancer treatment, practicing oncologists need to be aware of the potential risks of neurologic irAEs and be able to provide prompt treatment for this uncommon but potentially serious class of AEs. Treatment algorithms for relatively frequent AEs have been established, and there are many case reports of relatively frequent AEs that can be referenced $(14,15)$. The present case of aseptic meningitis is a rare AE, and the diagnosis and treatment of such events can be facilitated by the publication of detailed clinical case reports. Proper testing based on the careful observation of patients' clinical symptoms and intervention with steroids will enable the effective treatment at an earlier point in the clinical course.

\section{Acknowledgements}

Not applicable. 


\section{Funding}

No funding was received.

\section{Availability of data and materials}

All data generated or analyzed during the present study are included in this published article.

\section{Authors' contributions}

DT, NF and TN participated, conceived and designed the present case report, analyzed and interpreted the data and wrote the manuscript. DT, KI, TI and KT evaluated the patient and participated in therapy. $\mathrm{KI}$ and $\mathrm{MN}$ evaluated radiological images. TN and MN supervised the study and critically reviewed the manuscript. All authors have read and approved the final version of the manuscript.

\section{Ethics approval and consent to participate}

The present case report was approved by the Institutional Review Board of National Hospital Organization Kyushu Cancer Center (approval no. 2014-99). Written informed consent was obtained from the patient.

\section{Patient consent for publication}

The patient provided written informed consent for the publication of any associated data and accompanying images.

\section{Competing interests}

The authors declare that they have no competing interests.

\section{References}

1. Motzer RJ, Tannir NM, McDermott DF, Arén Frontera O, Melichar B, Choueiri TK, Plimack ER, Barthélémy P, Porta C, George $\mathrm{S}$, et al: Nivolumab plus ipilimumab versus sunitinib in advanced renal-cell carcinoma. N Engl J Med 378: 1277-1290, 2018.

2. Heng DY, Xie W, Regan MM, Warren MA, Golshayan AR, Sahi C, Eigl BJ, Ruether JD, Cheng T, North S, et al: Prognostic factors for overall survival in patients with metastatic renal cell carcinoma treated with vascular endothelial growth factor-targeted agents: Results from a large, multicenter study. J Clin Oncol 27: 5794-5799, 2009.

3. Heng DY, Xie W, Regan MM, Harshman LC, Bjarnason GA, Vaishampayan UN, Mackenzie M, Wood L, Donskov F, Tan MH, et al: External validation and comparison with other models of the international metastatic renal-cell carcinoma database consortium prognostic model: A population-based study. Lancet Oncol 14: 141-148, 2013.
4. Ardolino L and Joshua A: Immune checkpoint inhibitors in malignancy. Aust Prescr 42: 62-67, 2019.

5. Postow MA, Chesney J, Pavlick AC, Robert C, Grossmann K, McDermott D, Linette GP, Meyer N, Giguere JK, Agarwala SS, et al: Nivolumab and ipilimumab versus ipilimumab in untreated melanoma. N Engl J Med 372: 2006-2017, 2015.

6. Motzer RJ, Escudier B, McDermott DF, George S, Hammers HJ, Srinivas S, Tykodi SS, Sosman JA, Procopio G, Plimack ER, et al: Nivolumab versus everolimus in advanced renal-cell carcinoma. N Engl J Med 373: 1803-1813, 2015.

7. Hammers HJ, Plimack ER, Infante JR, Rini BI, McDermott DF, Lewis LD, Voss MH, Sharma P, Pal SK, Razak ARA, et al: Safety and efficacy of nivolumab in combination with ipilimumab in metastatic renal cell carcinoma: The CheckMate 016 study. J Clin Oncol 35: 3851-3858, 2017.

8. Hottinger AF: Neurologic complications of immune checkpoint inhibitors. Curr Opin Neurol 29: 806-812, 2016.

9. Tarhini A: Immune-mediated adverse events associated with ipilimumab ctla-4 blockade therapy: The underlying mechanisms and clinical management. Scientifica (Cairo) 2013: 857519, 2013.

10. Ibrahim RA, Berman DM, DePril V, Humphrey RW, Chen T, MessinaK M Chin M, Liu HY, Bielefield M and Hoos A: Ipilimumab safety profile: Summary of findings from completed trials in advanced melanoma. J Clin Oncol 29 (15 Suppl): S8583, 2011.

11. Caturegli P, Di Dalmazi G, Lombardi M, Grosso F, Larman HB, Larman T, Taverna G, Cosottini M and Lupi I: Hypophysitis secondary to cytotoxic T-lymphocyte-associated protein 4 blockade: Insights into pathogenesis from an autopsy series. Am J Pathol 186: 3225-3235, 2016.

12. Schadendorf D, Wolchok JD, Hodi FS, Chiarion-Sileni V, Gonzalez R, Rutkowski P, Grob JJ, Cowey CL, Lao CD, Chesney J, et al: Efficacy and safety outcomes in patients with advanced melanoma who discontinued treatment with nivolumab and ipilimumab because of adverse events: A pooled analysis of randomized phase II and III trials. J Clin Oncol 35: 3807-3814, 2017.

13. Spain L, Walls G, Julve M, O'Meara K, Schmid T, Kalaitzaki E, Turajlic S, Gore M, Rees J and Larkin J: Neurotoxicity from immune-checkpoint inhibition in the treatment of melanoma: A single centre experience and review of the literature. Ann Oncol 28: 377-385, 2017.

14. National Comprehensive Cancer Network: Guidelines on Management of Immune Checkpoint Inhibitor-Related Toxicities Version 2, 2019. https://www.nccn.org/professionals/physician_ gls/pdf/immunotherapy.pdf/. Accessed Jun 3, 2019.

15. Brahmer JR, Lacchetti C, Schneider BJ, Atkins MB, Brassil KJ, Caterino JM, Chau I, Ernstoff MS, Gardner JM, Ginex P, et al: Management of immune-related adverse events in patients treated with immune checkpoint inhibitor therapy: American society of clinical oncology clinical practice guideline. J Clin Oncol 36: 1714-1768, 2018.

This work is licensed under a Creative Commons Attribution-NonCommercial-NoDerivatives 4.0 International (CC BY-NC-ND 4.0) License. 